\title{
Impact of late administration of corticosteroids in COVID-19 ARDS
}

\author{
Nicolas Mongardon 1,2,3,4, Michael Piagnerelli ${ }^{5}$, David Grimaldi ${ }^{4,6}$, Bastien Perrot ${ }^{7}$ \\ and Jean-Baptiste Lascarrou ${ }^{4,8^{*}}$ on behalf of COVADIS study group investigators
}

C 2020 Springer-Verlag GmbH Germany, part of Springer Nature

\section{Dear Editor,}

Acute respiratory distress syndrome (ARDS) related to coronavirus disease 2019 (COVID-19) is challenging. As pro-inflammatory patterns and cytokine storm are among the hallmarks of severe COVID-19 pneumonia, early low dose of corticosteroids (CTC) has been proved to be effective in the RECOVERY trial [1] and subsequent meta-analysis [2]. However, a large proportion of patients remained dependent of mechanical ventilation at the end of the CTC therapy, and questions about late administration and possibly high doses of CTC to prevent or treat lung fibrosis in persistent ARDS [3] are unresolved. We, therefore, aimed to evaluate the impact of late CTC for COVID-19 ARDS patients versus the absence of CTC administration.

We performed a post hoc analysis from the COVADIS project, a multicenter observational study gathering 21 French and Belgian intensive care units (ICUs) [4]. All consecutive patients (from 10/03 to 15/04/2020) with moderate to severe ARDS according to Berlin definition under invasive ventilation and positive SARS-CoV-2 RT-PCR were included. We studied patients who did not receive (no CTC group) or received CTC later than 13 days after symptoms onset (75\% percentile of patients included in the RECOVERY trial [1]) (CTC group). We analyzed the effect of CTC administration on ICU 90-day survival with a Cox model by specifying the delay between intubation and administration of CTC as a time-dependent covariate. Potential

\footnotetext{
*Correspondence: Jeanbaptiste.lascarrou@chu-nantes.fr

${ }^{8}$ Médecine Intensive Réanimation, University Hospital Center of Nantes,

30 Boulevard Jean Monnet, 44000 Nantes Cedex 9, France

Full author information is available at the end of the article
}

The members of COVADIS study group investigators are listed in Acknowledgements section. confounding factors (age, gender, body mass index, hypertension, prone position, venovenous extracorporeal membrane oxygenation (VV-ECMO), comorbidities, static compliance and $\mathrm{PaO}_{2} / \mathrm{FiO}_{2}$ at intubation) were tested for inclusion in the final model. The same strategy was used to study the effect of CTC on mechanical ventilation duration, after censoring for death. The study was approved by appropriate regulatory French and Belgian committees, with the information of the patient or next of kin.

After exclusion of patients who: withdraw consent $(n=1)$, were included in other studies on CTC $(n=22)$, received early CTC $(n=24)$, or were lost to follow-up, 348 patients were finally analyzed.

Patients received a median dose of $1[1,2] \mathrm{mg} / \mathrm{kg}$ of methylprednisolone equivalent, 21 [18-26] days after symptoms onset (eSupplement). Delay between intubation and late initiation of CTC was 11 [8-16] days ( $n=57$ treated patients). In the final Cox model, late initiation of CTC was neither associated with lower ICU mortality $(\mathrm{HR}=1.44$; $95 \% \mathrm{CI}$ [0.83-2.50]) nor with shorter duration of mechanical ventilation $(\mathrm{HR}=0.89 ; 95 \% \mathrm{CI}[0.60-1.33]$ (Tables 1, 2).

Early CTC treatment has demonstrated survival benefit in severe COVID-19 pneumonia, and has been endorsed by WHO $[1,2]$. Conversely, no data are available on the impact of late CTC administration [5]. This post hoc analysis of a homogeneous cohort of the most severe critically ill patients found that late CTC administration did not improve the patient-centered outcomes.

To conclude, while early administration of low-dose CTC should be encouraged in severe COVID-19 pneumonia, late high-dose CTC appear to be non-beneficial in late non-resolving ARDS. Furthers trials are needed to better define the use of CTC in ARDS related to COVID-19.

\section{实


Table 1 Cox regression model for ICU 90-day mortality with late CTC administration as a time-dependent covariate

\begin{tabular}{llll} 
& Hazard ratio & $p$ value & 95\% confidence interval \\
Late CTC administration & 1.44 & 0.200 & $0.83-2.50$ \\
Age of patient & 1.08 & 0.000 & $1.04-1.11$ \\
Static compliance at intubation & 0.980 & 0.019 & $0.963-0.997$ \\
V-ECMO & 2.11 & 0.003 & $1.28-3.47$ \\
Charlson score & 1.17 & 0.000 & $1.08-1.25$ \\
Age $\times$ time $^{\text {a }}$ & 0.998 & 0.004 & $0.996-0.999$ \\
\hline
\end{tabular}

${ }^{a}$ Interaction term between age of patient and time (days)

Table 2 Cox regression model for MV release with late CTC administration as a time-dependent covariate

\begin{tabular}{llll} 
& Hazard ratio & $p$ value & $\begin{array}{l}95 \% \text { confidence } \\
\text { interval }\end{array}$ \\
\hline Late CTC administration & 0.89 & 0.577 & $0.60-1.33$ \\
Age of patient & 0.967 & 0.000 & $0.953-0.981$ \\
Prone position & 0.51 & 0.000 & $0.36-0.71$ \\
W-ECMO & 0.30 & 0.000 & $0.19-0.49$ \\
\hline
\end{tabular}

\section{Electronic supplementary material}

The online version of this article (https://doi.org/10.1007/s00134-020-06311-z) contains supplementary material, which is available to authorized users.

\section{Author details}

1 Service d'Anesthésie-Réanimation Chirurgicale, DMU CARE, DHU A-TVB, Assistance Publique-Hôpitaux de Paris (AP-HP), Hôpitaux Universitaires Henri Mondor, 94010 Créteil, France. ${ }^{2}$ Faculté de Santé, Univ Paris Est Créteil, 94010 Créteil, France. ${ }^{3}$ U955-IMRB, Equipe 03 "Pharmacologie et Technologies Pour les Maladies Cardiovasculaires (PROTECT)", Inserm, Univ Paris Est Creteil (UPEC), Ecole Nationale Vétérinaire d'Alfort (EnVA), 94700 Maisons-Alfort, France. ${ }^{4}$ AfterROSC Research Group, 75014 Paris, France. ${ }^{5}$ Intensive Care, CHU-CharleroiMarie Curie, Université Libre de Bruxelles, 140, Chaussée de Bruxelles, 6042 Charleroi, Belgium. ${ }^{6}$ Soins Intensifs, Hôpital Erasme, ULB, Route de Lennik 808, 1070 Bruxelles, Belgium. ${ }^{7}$ UMR_S 1246 Methods in Patient-Centered Outcomes and Health Research, Nantes University, 44000 Nantes, France. ${ }^{8}$ Médecine Intensive Réanimation, University Hospital Center of Nantes, 30 Boulevard Jean Monnet, 44000 Nantes Cedex 9, France.

\section{Acknowledgements}

COVADIS study group investigators: Nadia Aissaoui, MD; Medecine Intensive Réanimation, Hôpital Européen Georges Pompidou, Paris, France. Gauthier Blonz, MD; Medecine Intensive Reanimation, La Roche Sur Yon, France. Giuseppe Carbutti, MD; Unité de soins intensifs, CHR Mons-Hainaut, Mons, Belgium. Romain Courcelle, MD; Unité de soins intensifs, Centres Hospitaliers de Jolimont, La Louvière, Belgium. Stéphane Gaudry, MD; Réanimation médico-chirurgicale CHU Avicenne, Université Sorbonne Paris Nord, Bobigny France. Alain D'hondt, MD; Unité de soins intensifs, CHU Ambroise Paré, Mons, Belgium. Julien Higny, MD; Unité de soins intensifs, CHU Dinant Godinne, site Dinant, Belgium. Geoffrey Horlait, MD; Unité de soins intensifs, CHU Dinant Godinne, site Godinne, Belgium. Sami Hraiech, MD; Médecine Intensive Réanimation, Assistance Publique-Hôpitaux de Marseille, Hôpital Nord Marseille, France. Laurent Lefebvre, MD; Réanimation polyvalente, Centre Hospitalier du pays d'Aix, Aix en Provence, France. Francois Lejeune, MD; Unité de soins intensifs, Clinique Notre Dame de Grâce, Gosselies, Belgium. André Ly, MD; Service d'anesthésie-réanimation chirurgicale, Unité de réanimation chirurgicale polyvalente, Hôpitaux Universitaires Henri Mondor, Créteil. Zoe Pletschette, MD; Soins Intensifs, Hôpital Erasme, ULB, Route de Lennik 808, 1070 Bruxelles, Belgium. Bertrand Sauneuf, MD; Réanimation-Médecine Intensive, Centre Hospitalier Public du Cotentin, Cherbourg-en-Cotentin, France. Nicolas Serck, MD; Unité de soins intensifs, Clinique Saint Pierre, Ottignies, Belgium. Thibaud Soumagne,
MD; Médecine Intensive Réanimation, CHU Besançon, Besançon, France. Piotr Szychowiak, MD; Médecine Intensive Réanimation, CHRU Tours, Tours, France. Julien Textoris, MD, PhD; Laboratoire Commun de Recherche bioMérieux-Hospices Civils de Lyon-Université de Lyon 1, EA7426 PI3, Lyon, France. Benoit Vandenbunder, MD; Groupe des anesthésistes réanimateurs, Hôpital Privé d'Antony, Antony, France. Christophe Vinsonneau, MD; Service de Médecine Intensive Réanimation et Unité de Sevrage Ventilatoire et Réhabilitation Centre Hospitalier de Béthune, Beuvry, France.

Compliance with ethical standards

\section{Conflicts of interest}

All authors have no disclosures.

\section{Publisher's Note}

Springer Nature remains neutral with regard to jurisdictional claims in published maps and institutional affiliations.

Accepted: 22 October 2020

Published online: 6 November 2020

\section{References}

1. RECOVERY Collaborative Group, Horby P, Lim WS, Emberson JR, Mafham M, Bell JL, Linsell L, Staplin N, Brightling C, Ustianowski A, Elmahi E, Prudon B, Green C, Felton T, Chadwick D, Rege K, Fegan C, Chappell LC, Faust SN, Jaki T, Jeffery K, Montgomery A, Rowan K, Juszczak E, Baillie JK, Haynes R, Landray MJ (2020) Dexamethasone in hospitalized patients with Covid-19 - preliminary report. N Engl J Med. https://doi.org/10.1056/ NEJMoa2021436

2. WHO Rapid Evidence Appraisal for COVID-19 Therapies (REACT) Working Group, Sterne JAC, Murthy S, Diaz JV, Slutsky AS, Villar J, Angus DC, Annane $D$, Azevedo LCP, Berwanger O, Cavalcanti AB, Dequin PF, Du B, Emberson J, Fisher D, Giraudeau B, Gordon AC, Granholm A, Green C, Haynes R, Heming N, Higgins JPT, Horby P, Jüni P, Landray MJ, Le Gouge A, Leclerc M, Lim WS, Machado FR, McArthur C, Meziani F, Møller MH, Perner A, Petersen MW, Savovic J, Tomazini B, Veiga VC, Webb S, Marshall JC (2020) Association between administration of systemic corticosteroids and mortality among critically ill patients with COVID-19: a meta-analysis. JAMA. 324(13):13301341. https://doi.org/10.1001/jama.2020.17023

3. Steinberg KP, Hudson LD, Goodman RB, Hough CL, Lanken PN, Hyzy R, Thompson BT, Ancukiewicz M, National Heart L, Blood Institute Acute Respiratory Distress Syndrome Clinical Trials N (2006) Efficacy and safety of corticosteroids for persistent acute respiratory distress syndrome. N Engl J Med 354:1671-1684

4. Grimaldi D, Aissaoui N, Blonz G, Carbutti G, Courcelle R, Gaudry S, Gaultier A, D'Hondt A, Higny J, Horlait G, Hraiech S, Lefebvre L, Lejeune F, Ly A, Piagnerelli M, Sauneuf B, Serck N, Soumagne T, Szychowiak P, Textoris J, Vandenbunder B, Vinsonneau C, Lascarrou JB, Group Cs (2020) Characteristics and outcomes of acute respiratory distress syndrome related to COVID-19 in Belgian and French intensive care units according to antiviral strategies: the COVADIS multicentre observational study. Ann Intensive Care 10:131 
5. Arulkumaran N, Snow TAC, Longobardo A, Brealey D, Singer M (2020) Steroids in ARDS: more light is being shed. Intensive Care Med. 4:1-3. https://doi.org/10.1007/s00134-020-06230-z 